\title{
Efficient Channelization for PMR+4G and GSM Re-Farming Base Stations
}

\author{
Álvaro Palomo Navarro, Rudi Villing, Ronan Farrell
}

\author{
Callan Institute, Department of Electronic Engineering \\ National University of Ireland, Maynooth \\ email : apalomo@eeng.nuim.ie,rvilling@eeng.nuim,rfarrell@eeng.nuim.ie
}

\begin{abstract}
Current trends in mobile communications look for a better usage of the frequency spectrum by diverging from the classic frequency bands division for each standard. Instead, sharing a same frequency band by several mobile standards has been motivated by several factors: under-utilisation of some frequency bands, better electromagnetic propagation properties and provision of new capabilities to existing standards. This new way to manage the electromagnetic spectrum has an influence in the devices which form the mobile radio interface: base stations and mobiles stations. In particular for base stations, channelization represents an important challenge. In this paper efficient channelization techniques are proposed as a practical solution for real world professional and commercial mobile communication cases where frequency bands are shared. Depending on each case, the most optimal solution is based on the application of one of these channelization techniques, or a combination of several of them.
\end{abstract} re-farming

Keywords - Dynamic spectrum allocation, non-uniform channelization, PMR+4G, GSM

\section{INTRODUCTION}

In wireless communications, spectrum has traditionally been divided into different coarse frequency bands each of which is allocated to just one wireless standard. The alternative to this is frequency band multiplexing in which a set of different communication standards may share a single frequency band. In its most flexible form, Dynamic Spectrum Allocation (DSA), more efficient utilisation of the available radio frequency spectrum can be achieved. In particular, DSA offers a solution to the under-utilisation of frequency bands reserved for standards with a low data traffic demand or which do not require a 24 hour usage by sharing them with standards with a higher traffic demand.

The possibility of sharing a frequency band between multiple standards has been considered separately for both private/professional and commercial mobile communication standards. In the field of Professional Mobile Radio (PMR), the data rates offered by Terrestrial Trunked Radio (TETRA) and its high-speed evolution, TETRA Enhance Data Service (TEDS) [1] are not sufficient for advanced PMR applications such as remote patient monitoring, full-duplex video streaming, advanced telemetry, mobile robot control, 3D localization, and geographical information systems. To address this it has been proposed that a fourth generation (4G) broadband wireless technology-either Worldwide
Interoperability for Microwave Access (WiMAX) or Long Term Evolution (LTE)—would be integrated with TETRA/TEDS [2]. Furthermore, the integration should not require additional spectrum allocations.

In the commercial communications field, frequency band multiplexing has been considered to allow the reuse or re-farming of the Global System for Mobile Communications (GSM) 900 and $1800 \mathrm{MHz}$ frequency bands with third and fourth generation mobile communication standard channels such as the Universal Mobile Telecommunications Standard (UMTS) [3-4]. The main objective of re-farming the GSM900 frequency band is to bring broadband communications to rural areas with low population density. Because the GSM900 and GSM1800 bands have lower carrier frequencies than the general UMTS frequency band around $2.1 \mathrm{GHz}$, lower path losses are experienced and cell sizes can be up to 2.5 times larger than UMTS2100. Consequently the number of base stations required to cover an area may be reduced.

Although DSA can help to solve the spectrum under-utilisation, it does require some changes in the radio frequency interface between the base stations and the mobile stations. In a DSA implementation, the physical channels of the multiple standards which now share a frequency band are multiplexed onto a single downlink or uplink signal. Furthermore, channels belonging to different standards can (and usually do) have different bandwidths and centre frequency allocation requirements. In channelization terms, the 


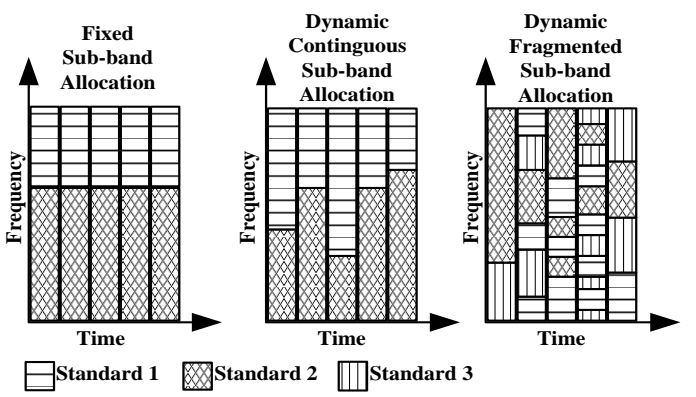

Figure 1 Different DSA configurations [5].

extraction of these non-uniform types of channels at the base station requires a non-uniform channelizer to filter them at the required centre frequencies. Since DSA implies dynamic reallocation of spectrum to different standards over time, the non-uniform channelizer must be dynamically reconfigurable. For this reason, two of the most desirable characteristics of a non-uniform channelizer are channel bandwidth flexibility and reconfigurability.

\section{SOFTWARE DEFINED RADIO BASED RECEIVER FOR DSA}

Frequency band sharing between several standards can be achieved by either fixed or dynamic allocation of the standard channels into sub-bands [5], as depicted in Figure 1. Each one of the three schemes in Figure 1 represents a possible allocation scheme for the common uplink or downlink signal. If Fixed Sub-band Allocation (FSA) is used, then fixed non-overlapping ranges of the shared frequency band are reserved for each standard. This scheme, which is essentially the traditional independent frequency bands configuration applied at a finer granularity, is the least flexible but simplest to implement option.

In contrast, DSA schemes are more flexible and permit better spectrum utilization (because fixed sub-bands need not be reserved when not in use). Two DSA schemes are considered here and represented in Figure 1: Dynamic Contiguous Sub-band Allocation (DCSA) and Dynamic Fragmented Sub-band Allocation (DFSA). DCSA allocates standards to adjacent frequency sub-bands but does not constrain the dividing frequency between them. If one sub-band is under-utilised then the dividing frequency may be moved to expand the bandwidth (and capacity) of an adjacent band. However, the limitations and complexity of this scheme increase when more than two standards must share the frequency band.

In DFSA, the most flexible scheme, each standard is allocated different bandwidth fragments within the shared frequency band depending on its traffic needs. The bandwidth of these fragments can range from a single channel to the whole frequency band (if, for example, only one of the standards needed to allocate channels at that specific instant). Unlike DCSA, a standard may be allocated multiple fragments and these fragments need not be contiguous.

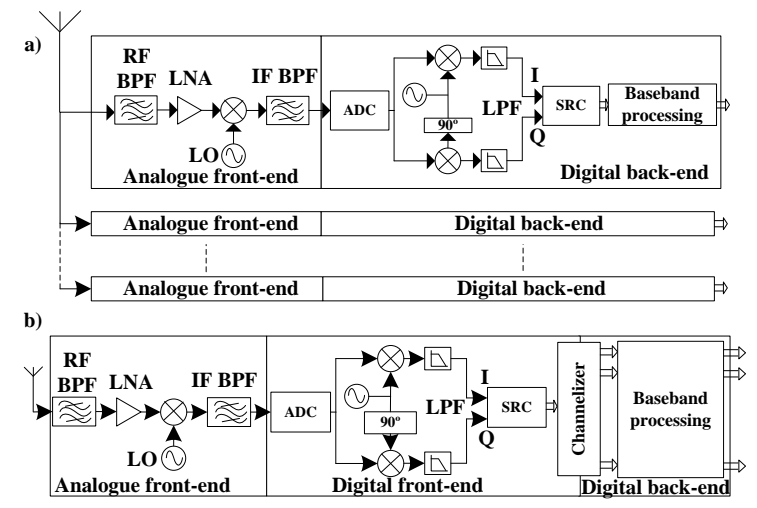

Figure 2 Base station receiver, a) Hardware based b) SDR based.

Traditionally, a base station receiver is composed of parallel hardware blocks, each containing dedicated circuitry to handle a single channel. Figure 2 a shows this structure and how each channel is independently filtered and down-converted from the received RF signal. In each branch the analogue front-end is responsible for filtering the channel of interest from the other channels in the UL signal and down-converting it. Subsequently, the digital back-end performs the digital baseband operations. Furthermore the per-channel circuits are usually designed to handle just one type of communication channel. If the base station supports more than one mobile communication standard, more than one type of receiver structure is employed for as many channels as each standard requires.

The suitability of the structure in Figure $2 \mathrm{a}$ for DSA schemes varies depending on whether FSA, DCSA or DFSA channel allocation is considered (see Figure 1). For FSA, the hardware based per-channel receiver is a reasonable solution since neither the number of channels nor their centre frequencies and bandwidths vary in the FSA scheme. However, when DCSA or DFSA schemes are considered, the use of dedicated hardware circuits for each channel is more complex (and less efficient) since every possible channel allocation configuration has to be implemented. This becomes especially impractical for DFSA.

In a Software-Defined Radio (SDR) receiver (Figure 2b) one single Analogue-to-Digital Converter (ADC) is placed as close as possible to the antenna. Located in the digital front-end [6] the ADC digitizes the frequency band containing all the channels of interest at once rather than digitizing each channel independently. Subsequently, a channelizer extracts the independent information channels.

To make SDR reconfigurable the digital front-end and back-end are usually implemented on programmable hardware platforms such as Field Programmable-Gate Arrays (FPGA) and General Purpose Processors (GPP). In general programmable hardware sacrifices efficiency for flexibility and this makes it challenging to realize SDR systems in practice. In particular for multi-standard base stations, 
the non-uniform channelization of a large number of channels from different standards is a computationally expensive operation to perform [6]. This task becomes even more complex if DSA schemes are supported, especially DFSA.

\section{EFFICIENT NON-UNIFORM CHANNELIZATION FOR DSA BASE STATIONS}

Non-uniform channelization techniques for SDR devices have been widely proposed in literature [7-8]. In general, the designs fall into two categories: complex modulated filter banks, where part of the computation required in the channels separation is shared by all the channels, and per-channel designs where each channel is independently filtered and computation is not shared across channels [9].

\section{a) Complex Filter Bank Based Channelizers}

In communications, modulated filter banks (also known as transmultiplexers in this context) have been widely used due to the low sample rate at which the filtering operations are performed and hence their relatively low complexity and high efficiency [10]. For wireless communications in particular, baseband signal processing is carried out using complex valued signals. For this reason, complex modulated filter banks such as the Discrete Fourier Transform modulated Filter Banks (DFT-FB) and Exponential Modulated Filter Banks (EMFB) are employed. Both types can be obtained from a more general uniform modulated filter bank known as Generalized DFT modulated Filter Bank (GDFT-FB) [10].

A GDFT-FB on its own can only implement a uniform channelizer (for channels whose bandwidth and channel spacing characteristics are all identical). To implement a non-uniform channelizer, two approaches are considered: the Parallel GDFT-FB (PGDFT) and the Recombined GDFT-FB (R-GDFT) [8]. These are shown in Figure 3. In the P-GDFT, several GDFT-FBs in parallel process the wideband signal. A frequency band shared by $J$ standards or channel types (having different channel bandwidth and centre frequency allocations) is channelized using $J$ parallel GDFT-FBs. In contrast the R-GDFT uses just a single GDFT-FB, but adds recombination blocks at the outputs to construct wider channels by recombining GDFT-FB output sub-bands.

Using the P-GDFT (Figure 3a), each individual GDFT-FB outputs the complete set of $K_{j}$ channels for standard $j$. Any DFSA configuration of channel types can be channelized simply by selecting appropriate outputs from each GDFT-FB. Changes in the DFSA configuration do not require redesign or re-optimization of the channelizer structure. Only the selection of GDFT-FB outputs needs to be adapted. An upgrade that introduces a new channel type (whose characteristics are not met by any of the existing filter banks) would, however, require the introduction of a new GDFT-FB in parallel with the others.

DFSA channelization using the R-GDFT (Figure $3 \mathrm{~b})$ is achieved by recombining the $R_{j}$ contiguous

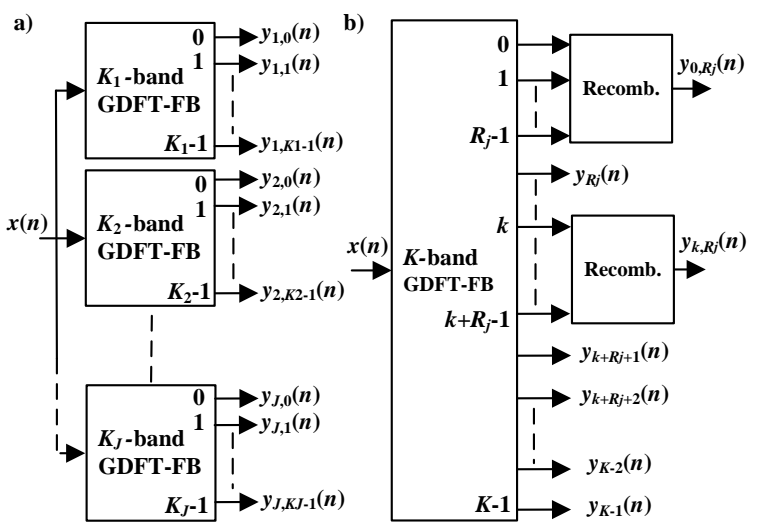

Figure 3: GDFT-FB based non-uniform channelizers for $\mathbf{J}$ $(\mathrm{j}=1, \ldots, \mathrm{J})$ different standards a) P-GDFT b) R-GDFT.

GDFT-FB output sub-bands required for each channel of standard $j$, or directly selecting appropriate GDFTFB output sub-bands in the case that standard $j$ requires no recombination. The bandwidth of the GDFT-FB sub-bands is the granularity bandwidth used to divide up the wideband input frequency band. It may be chosen equal to the narrowest bandwidth channel to be extracted (e.g. $25 \mathrm{kHz}$ for TETRA/TEDS networks as in [9]) or a narrower granularity bandwidth may be chosen. A narrower bandwidth relaxes the bandwidth and centre frequency constraints across channel types. It does, however, increase the number of sub-bands that must be recombined for each channel (and therefore the number of operations required). An upgrade that introduces a new channel type may be handled by increasing the number of sub-bands to be recombined unless the new channel bandwidth is not a multiple of the existing granularity band (in which case the granularity band would need to be readjusted).

Comparing both approaches, the R-GDFT offers more flexibility in terms of centre frequencies, but it can require more operations than the P-GDFT when the input wideband signal is mainly occupied by relatively wide channels. For both structures, very efficient filter designs and fewer operations can be obtained by applying multi-stage filtering techniques [8].

\section{b) Farrow Per-Channel Channelizers}

Farrow filters may be used to synthesize a fractional controllable delay or carry out an arbitrary sample rate conversions (SRC) by using polynomialbased interpolation [11]. This is especially useful when applied to irrational SRC factors. Internally, the Farrow filter is formed by a set of sub-filters whose coefficients are multiplied by the set of fractional delay values $(\tau)$. When the SRC factor must be changed, only the $\tau$ values need to be adapted, leaving the filter coefficients fixed.

A Farrow Per-Channel Channelizer (FPCC) is implemented by connecting several Farrow filters in parallel [12], as shown in Figure 4. In each branch the input wideband signal is first shifted to centre the appropriate channel at baseband using a mixer. 


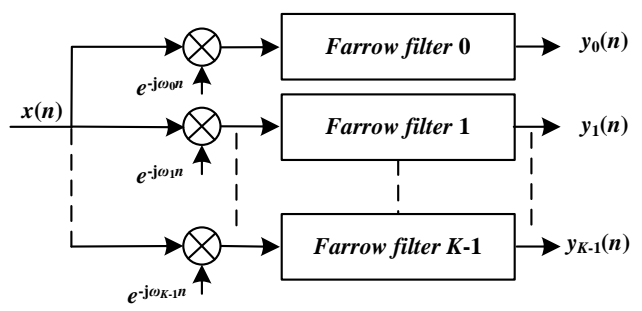

Figure 4: FPCC structure.

Thereafter, the baseband channel is isolated via a lowpass Farrow filter. Therefore, each branch can be independently reconfigured to extract a single channel of standard $j$ by modification of the mixer frequency and the Farrow filter $\tau$ values for that branch. The total number of branches, $K$, is simply the maximum number of channels that would need to be simultaneously channelized. Generally this corresponds to the number of smallest bandwidth channels that fully occupy the frequency band.

To channelize an arbitrary DFSA configuration, each of the desired channels is first assigned to a branch of the FPCC (permitting some branches to be disabled if fewer than the maximum number of channels is required). Thereafter, in each branch, the frequency mixer is tuned to the required centred frequency and the appropriate $\tau$ values are loaded into the Farrow filter to perform the required SRC.

If the FPCC needs to be upgraded to support a new standard, two actions need to be performed. First, the calculation of the filter $\tau$ values to perform the required filtering and SRC for the new standard channels. Second, only if the new standard has a bandwidth smaller than any other already supported, new branches in parallel need to be added to support the worse case allocation scenario.

\section{c) R-GDFT and P-GDFT vs. FPCC}

The FPCC is more flexible than the GDFT-FB based designs in terms of channel bandwidths and centre frequencies because each channel is independently processed and because irrational SRC factors can be used. However, it has several disadvantages. First, there is the number of parallel processing paths that must be implemented to simultaneously extract a large number of channels (e.g. 200 in a typical TETRA V\&D band). Second, the filtering operations in each branch of the FPCC are performed at the high sample rate of the wideband input signal unlike the modulated filter banks which perform their filtering at a lower sample rate. Finally, unlike filter banks, there is no sharing of computation between channels. For these reasons, the FPCC is generally more suitable for channelizers that extract a small number of channels and less suitable for base stations which need to simultaneously extract a large number of channels embedded in the uplink signal.

The computational load of the R-GDFT, P-GDFT and FPCC was evaluated in [9] considering three different DFSA configurations of TETRA V\&D $(25 \mathrm{kHz})$, TEDS $50 \mathrm{kHz}$, and TEDS $100 \mathrm{kHz}$ channels sharing the $5 \mathrm{MHz}$ uplink TETRA band between 380 and $385 \mathrm{MHz}$. The results showed that the number of real multiplications per complex input sample was around three orders of magnitude larger for the FPCC than the GDFT-FB based channelizers. In general, the R-GDFT required between 10 and 16 percent fewer multiplications than the P-GDFT.

\section{Channelizer Design CASE Studies}

In this section, the design of channelizers for current trends in mobile communications, specifically PMR + $4 \mathrm{G}$ and GSM re-farming, is examined. It will be shown that it is not possible to recommend a single "best" channelizer structure-combinations of techniques sometimes provide the best solution.

\section{a) $P M R+4 G$}

Integration of $4 \mathrm{G}$ standards such as WiMAX and LTE can provide TETRA with the necessary high data transmission rates necessary for services such as live video streaming [2]. Both $4 \mathrm{G}$ standards are based on multicarrier Orthogonal Frequency Division Multiplexing (OFDM) techniques [13]. Mobile WiMAX (IEEE 802.16e) provides end-user mobility, variable channel bandwidths from $1.25 \mathrm{MHz}$ to 20 $\mathrm{MHz}$, and a theoretical peak data rate of $75 \mathrm{Mbps}$ (DL) / $25 \mathrm{Mbps}$ (UL). LTE, conceived as a long term evolution of UMTS, is the choice of the ETSI/3GPP for $4 \mathrm{G}$ packet-based commercial mobile communications. It defines six possible channel bandwidths between 1.4 and $20 \mathrm{MHz}$ and has a maximum data rate of 326.4 Mbps (DL) / 86.4 Mbps (UL).

Any update for broadband PMR base stations (integrating 4G) would in general have to maintain backward compatibility with legacy mobile stations. For legacy mobile terminals, the allocation of TETRA and TEDS channels using FDD and TDM in the DL and UL signals must remain the same. Furthermore, their centre frequencies must remain compliant with the ECC specification for PMR systems [14].

WiMAX and LTE can both operate in either TDD or FDD mode. By applying DSA configurations, two broadband channels, one in the UL and one in the DL, can be introduced in the TETRA frequency band using the FDD or TDD operation mode. Considering the internationally reserved TETRA frequency band between 380 and $400 \mathrm{MHz}$ up to three WiMAX 1.25 $\mathrm{MHz}$ or LTE $1.4 \mathrm{MHz}$ channels could be allocated in a $5 \mathrm{MHz}$ DL or UL band. The permanent reservation of bandwidth for one or more 4G channels (using an FSA scheme) would significantly reduce the TETRA/TEDS capacity and overall spectrum utilization. Therefore, it is better to use a DSA scheme so that the $4 \mathrm{G}$ channels could be used on an as-needed basis for those services which require the highest data rates.

Figure 5a shows a possible channel allocation for TETRA with a single $1.25 \mathrm{MHz}$ WiMAX or $1.4 \mathrm{MHz}$ 


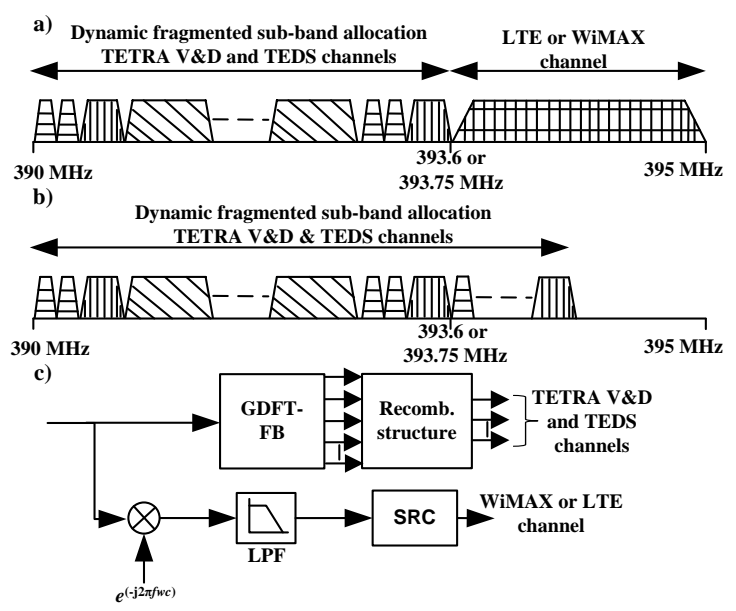

Figure 5: TETRA + WiMAX 1.25 MHz or LTE $1.4 \mathrm{MHz}$ channel a) DCSA configuration b) Efficient channelization structure.

LTE channel. This allocation scheme preserves more than two thirds of TETRA/TEDS capacity even when the $4 \mathrm{G}$ channel is in use. Due to the bandwidth difference between the broadband channel and the TETRA/TEDS channels and the allocation of just one possible broadband channel, a DCSA scheme is most appropriate. Therefore, when it is in use, the broadband channel is allocated a fixed centre frequency at one end of the frequency band. When the broadband channel is not required, the entire frequency band is available for TETRA and TEDS channel allocation, as shown in Figure 5b. Since the main use of TETRA V\&D and TEDS networks is for safety and security services, which must be robust in extreme situations, the availability of high priority channels must be immediate when required. If it is decided that the broadband service is primary, then the broadband channel bandwidth must be available on demand, immediately clearing any TETRA/TEDS channel occupying that bandwidth at that moment (by dropping calls or connections). On the other hand, if the TETRA channels are primary, then the broadband channel bandwidth should be cleared immediately if the demand for TETRA/TEDS channels exceeds the available capacity.

The efficient non-uniform channelization of TETRA V\&D and TEDS channels has been demonstrated in [8] using R-GDFT and P-GDFT structures with the R-GDFT exhibiting lower computational load [8]. To apply the R-GDFT to the proposed PMR+4G scheme, the R-GDFT for TETRA/TEDS would need to be extended with a recombination block for the broadband channel. However, the size of the required recombination (up to 56 of $25 \mathrm{kHz}$ granularity bands) suggests that R-GDFT alone is not an optimum solution. Instead, Figure $5 \mathrm{c}$ shows a more efficient solution in which the broadband channel is processed independently of the TETRA/TEDS channels. In this design the R-GDFT only deals with the TETRA V\&D and TEDS channels, whereas, only when the broadband channel is active, the parallel branch down-converts and filters the broadband channel independently.

\section{b) Re-Farming of GSM Bands}

So called re-farming of the GSM frequency bands has been studied as a possibility for providing additional spectrum with longer propagation distances to $\mathrm{GSM}$ based $3 \mathrm{G}$ and $4 \mathrm{G}$ communications standards. Significant effort has been focused on the deployment of $5 \mathrm{MHz}$ UMTS and HSPA channels in the GSM 900 and $1800 \mathrm{MHz}$ bands, an effort generally known as UMTS900 and UMTS1800 [3-4]. In addition, the deployment of LTE channels in the GSM1800 band has been also considered for the same reason as UMTS900. This alternative is generally known as LTE1800. The GSM1800 band provides a wider range of frequencies than GSM900, therefore allowing the use of larger LTE channel bandwidths.

For the particular case of the $900 \mathrm{MHz}$ band, a minimum of $7.5 \mathrm{MHz}$ is the bandwidth estimated that a mobile operator must posses in order to use the GSM900 + UMTS900 implementation [3]. In general, the bandwidth available is around $10 \mathrm{MHz}$. For this reason, the use of a single UMTS, HSPA or LTE (3 or $5 \mathrm{MHz}$ ) channel is considered. As an alternative, up to three 1.4 MHz LTE channels could be deployed. Mobile operators generally own larger bandwidths in the $1800 \mathrm{MHz}$ band, and this band is generally preferred for re-farming with the larger LTE channels.

Considering the $900 \mathrm{MHz}$ band, there is a difference between allocating the broadband channel at the edge of the operator frequency band or in the middle of it. If the channel is allocated at the edge, adjacent to another operator's bandwidth, a larger guard band is required [3]. For this reason, Figure 6a shows the $3 \mathrm{G}$ and $4 \mathrm{G}$ channels allocated in the middle of the $10 \mathrm{MHz}$ band reserved for an operator in the GSM900 band. Unlike PMR systems where strict prioritisation of channel types applies, in a commercial system a best effort approach could be used. Therefore, broadband channels would be allocated only if sufficient contiguous bandwidth was available.

Two channelizer designs which can handle the DFSA channel allocation schemes in Figure 6a (supporting one to three broadband channels) are considered here. Since UMTS, HSPA and LTE are all based on GSM (their bandwidths and possible centre frequencies are multiples of $200 \mathrm{kHz}$ ), it is possible to use a single R-GDFT to cover the entire $10 \mathrm{MHz}$ band with a granularity band equal to one GSM channel as shown in Figure 6b. Using the R-GDFT, any re-farmed broadband channel is extracted by recombining a number of uniform sub-bands. As an alternative to the R-GDFT design, a parallel structure consisting of a uniform GDFT-FB in one branch with per-channel channelizers in the remaining branches is shown in Figure 6c. In this design, the GDFT-FB channelizes the GSM channels. The first per channel branch is a reconfigurable FPCC able to channelize one $5 \mathrm{MHz}$ 


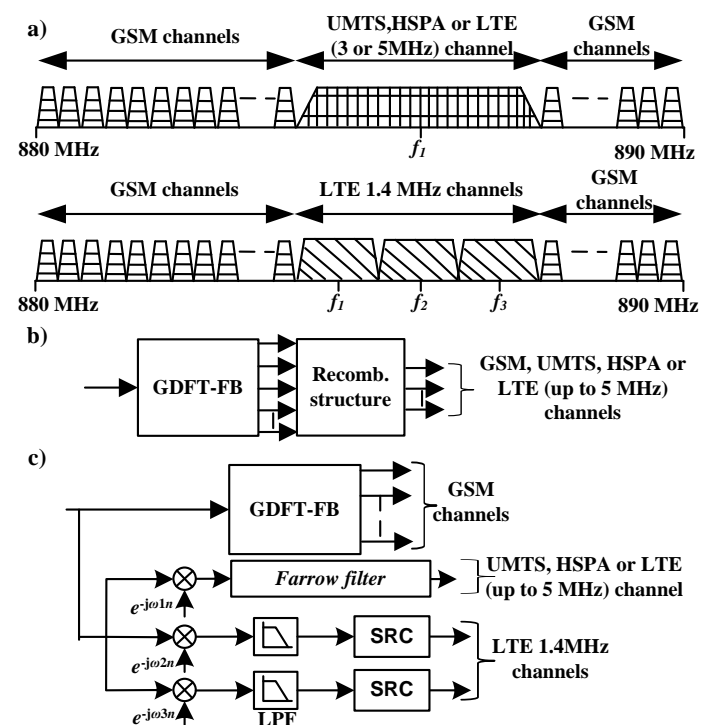

Figure 6: GSM $900 \mathrm{MHz}$ band re-farming a) Channel allocation, b) Channelizer option based on R-GDFT, c) Channelizer option based on GDFT-FB and FPCC in parallel.

UMTS, $3 \mathrm{MHz}$ LTE, or $1.4 \mathrm{MHz}$ LTE channel. The remaining two branches are not reconfigurable (except for centre frequency) and are required only when three 1.4 MHz LTE channels are allocated.

Based on the design guidelines given in [9], Figure 7 presents the number of real multiplications per input complex sample to the channelizer for both channelizer designs. Five variants of the DFSA scheme shown in Figure $6 \mathrm{a}$ are considered, differing only by the number of broadband channels allocated in the GSM frequency band. In general, it can be seen that the parallel channelizer (Figure 6c) requires fewer operations than the R-GDFT (Figure 6b) in all cases except when three LTE $1.4 \mathrm{MHz}$ channels are allocated. Furthermore, the difference in computational load between designs is most pronounced when a single wide UMTS channel is allocated (in which case just one of the per channel branches in the parallel channelizer is required). Therefore, the parallel design is more efficient for the DFSA scheme considered. It is worth noting, however, that this efficiency comes at the expense of flexibility: the R-GDFT in Figure $6 \mathrm{~b}$ would not generally require redesign if future re-farming added more broadband channels whereas the parallel channelizer would require additional parallel branches.

\section{$\mathrm{V} \quad$ CONCLUSIONS}

In this paper non-uniform channelization techniques were applied to current trends in mobile communications related to DSA. Previous publications [8] have proven that R-GDFT channelizers are the best option when there are a large number of channels of different bandwidths. In contrast, this work shows that when the channel bandwidths differ dramatically between standards sharing a frequency band, a parallel combination of the R-GDFT and per channel

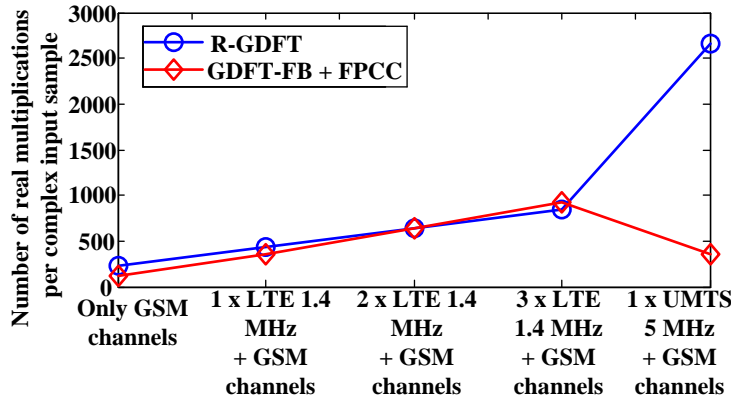

Figure 7: Computational load for the structures in Figure $6 \mathrm{~b}$ and Figure $6 \mathrm{c}$ for different DFSA schemes.

channelizers (including the FPCC) could be more effective.

\section{REFERENCES}

[1] M. Nouri, et al., "TEDS: A high speed digital mobile communication air interface for professional users," Vehicular Technology Magazine, IEEE, vol. 1, pp. 3242, 2006.

[2] A. Durantini, et al., "Integration of Broadband Wireless Technologies and PMR Systems for Professional Communications," in Networking and Services, 2008. ICNS 2008. Fourth International Conference on, 2008, pp. 84-89.

[3] H. Holma, et al., "UMTS900 Co-Existence with GSM900," in Vehicular Technology Conference, 2007. VTC2007-Spring. IEEE 65th, 2007, pp. 778-782.

[4] A. Moral, et al., "Assessment of the benefits of introducing a HSDPA carrier at $900 \mathrm{MHz}, "$ in GLOBECOM Workshops (GC Wkshps), 2010 IEEE, 2010, pp. 834-838.

[5] P. Leaves, et al., "Dynamic spectrum allocation in composite reconfigurable wireless networks," Communications Magazine, IEEE, vol. 42, pp. 72-81, 2004.

[6] P. B. Kenington, RF and Baseband Techniques for Software Defined Radio: Artech House, Inc. Mobile Communication Series, 2005.

[7] R. Mahesh, et al., "Filter Bank Channelizers for MultiStandard Software Defined Radio Receivers," Journal of Signal Processing Systems, Springer New York, 2008.

[8] A. Palomo Navarro, et al., "Practical Non-Uniform Channelization for Multi-standard Base Stations," ZTE Comms. Journal. Special topic: Digital Front-End and Software Radio Frequency in Wireless Communication and Broadcasting, vol. 9, December 2011.

[9] A. Palomo Navarro, "Channelization for Multi-Standard Software-Defined Radio Base Stations," PhD, Electronic Engineering Dept., National University of Ireland, Maynooth, 2011.

[10] R. E. Crochiere and L. R. Rabiner, Multirate Digital Signal Processing: Englewood Cliffs (NJ): Prentice Hall, 1983.

[11] C. W. Farrow, "A continuously variable digital delay element," in Circuits and Systems, 1988., IEEE International Symposium on, 1988, pp. 2641-2645 vol.3.

[12] A. Eghbali, et al., "A Multimode Transmultiplexer Structure," Circuits and Systems II: Express Briefs, IEEE Transactions on, vol. 55, pp. 279-283, 2008.

[13] T. Bhandare, "LTE and WiMAX Comparison," MSc, Santa Clara University, 2008.

[14] ECC, "Planning criteria and coordination of frequencies in the land mobile service in the range $29.7-921 \mathrm{MHz}$, T/R 25-08," 2008. 